\title{
Tendencias de consumo y de la distribución en el mercado de las arepas de maíz en la ciudad de Bogotá, D. C.*
}

\section{Consumption and distribution trends in the market of maize arepas}

in the city of Bogotá, D.C.

\author{
Campo Elías López Rodríguez** \\ Belkyz Adriana Moreno Luengas*** \\ Édgar Lombana Díaz****
}

Recibido: 17 de abril de 2017

Revisado: 18 de mayo de 2017

Aprobado: 31 de mayo de 2017

\section{Resumen}

La caracterización del mercado de las arepas de maíz en la capital colombiana puede generar inquietudes, pues elementos como las tendencias de consumo y los canales de distribución harán un aporte significativo al sector productivo de la ciudad que esté interesado en esta actividad económica. Su representación en la canasta familiar lo hace

* Artículo de investigación. DOI: http://dx.doi.org/10.15332/s0124-3551.2017.0030.06

** Magíster en Gerencia Estratégica de Mercadeo. Docente del programa de Comercio Internacional de la Universidad ECGI (Bogotá, Colombia). Correo de contacto: clopezr@ecci.edu.co. http://orcid.org/0000-0003-4061-2979

*** Magíster en Dirección de Empresas Multinacionales, Universidad Autónoma de Madrid. KAM Canal Ladrillera Santafé. Correo de contacto: belkyza7@hotmail.com 
accesible a todos los niveles socioeconómicos, pues existen diferentes formatos y precios que el consumidor puede elegir. $\mathrm{Al}$ respecto, en cuanto a la metodología, en la primera parte del trabajo de campo se elaboró un estudio basado en un diseño descriptivo de tipo cuantitativo, empleando un muestreo no probabilístico con 384 unidades de información; en la segunda parte se desarrolló una observación de carácter cualitativo utilizando bitácoras para la recolección de la información. Los resultados del estudio determinan rasgos representativos que caracterizan las tendencias de consumo y de los canales de distribución presentes en el mercado de la arepa de maíz en la ciudad de Bogotá, D. C.

Palabras clave: Mercadeo, consumo, canales de distribución, mercados.

Clasificación JEL: M10, M30, M31.

\section{Abstract}

The characterization of the maize arepas market in the Colombian capital can generate concerns, since elements such as consumption trends and distribution channels will make a significant contribution to the city's productive sector that is interested in this economic activity. Its representation in the market basket makes it accessible to all socioeconomic levels, since there are different formats and prices that the consumer can choose. Regarding the methodology, in the first part of the fieldwork a study was elaborated based on a descriptive design of quantitative type, using a non-probability sampling with 384 information units; in the second part, a qualitative observation was developed using logbooks to collect the information. The results of the study determine representative features that characterize the consumption trends and the distribution channels present in the maize arepa market in the city of Bogotá, D.C.

Keywords: Marketing, consumption, distribution channels, markets.

Classification JEL: M10, M30, M31. 


\section{Introducción}

En los actuales estudios de marketing, las tendencias de consumo son un elemento relevante en la estructura no solo de los modelos de negocios, sino también de las ofertas empresariales de aquellas organizaciones que poseen un enfoque dirigido hacia el mercado. Por ello, Hernández y Vega (2017) mencionan que "es indispensable para el ámbito empresarial analizar y conocer la población actual, las tendencias, sus comportamientos, sus necesidades y deseos para poder crear estrategias mercadológicas que satisfagan estos aspectos" (p. 728).

Las empresas requieren contar con una serie de conocimientos sobre sus consumidores que les permitan optimizar los procesos estratégicos, tácticos y operativos en relación con su actividad de marketing, persiguiendo el crecimiento de vínculos no solo transaccionales con los clientes, sino de aquellos de tipo relacional e incluso sensorial (Ortegón-Cortázar y Gómez, 2016). París (2015) propone que si se pretende estudiar el comportamiento del consumidor se deben tener en cuenta dos elementos: la mente del consumidor, entendida esta como la conjugación y expresión de una serie de circunstancias propias del mundo moderno particularmente complejas y estrechamente relacionadas con el acto y la acción de adquisición y uso de productos (Páramo, 2004), y la mente del mercado, que no solo condiciona, sino que hace que el consumidor cambie su conducta de compra cuando su consumo está influenciado por los otros. El análisis de las tendencias de consumo debe ser una prioridad para los mercadólogos, pues estas cambian constantemente y se van adaptando a las nuevas dinámicas del comportamiento humano (Hernández y Vega, 2017).

Una de las actividades del marketing mix que potencializa el consumo en términos de disponibilidad de productos es el proceso de distribución, el cual se fundamenta en el canal de marketing, que es el "conjunto de actividades que permite el traslado de productos desde su lugar de fabricación hasta el establecimiento comercial, y actúa como puente entre el sector productivo y el del consumo" (Ares y Brenes, 2014, p. 25). Todos los procesos de innovación que se relacionen con la distribución agregarán un alto contenido de valor a las ofertas empresariales (Rebollo y Casares, 2002), por esta razón, la gestión de la distribución es parte fundamental de las actividades de marketing táctico de las organizaciones, debido a que, "ante la existencia de un flujo físico de mercancías, es preciso también considerar las decisiones que integran el proceso de distribución física, es decir, procesamiento de pedidos, organización y localización de almacenes, gestión de existencias, medios de transporte, etc." (Peris, Parra, Lhermie y Romero, 2008, p. 93). Esta actividad facilita la penetración y el desarrollo de nuevos mercados, es decir, crecimiento empresarial y comercial; por ello, el hecho de que los mercados actuales sean volátiles y dinámicos, desde su componente psicológico hasta su entorno, hace que la red de distribución también deba evolucionar y diversificarse de una forma paralela (López Rodríguez, 2016). 
Con base en lo expuesto, el presente estudio tiene como objetivo reconocer las tendencias de consumo y de la distribución en el mercado de las arepas de maíz en la ciudad de Bogotá, D. C., por ser este producto una alternativa relevante en la canasta familiar capitalina, y más aún cuando, según un reciente estudio elaborado por Nielsen -empresa líder mundial en medición de hábitos de consumo-, el 73 \% de los colombianos incluye una arepa de maíz en el desayuno (Portafolio, 2016). Para lograrlo, se desarrolló una metodología en la que inicialmente de aplicó un diseño descriptivo de tipo cuantitativo por medio de una encuesta tanto a hombres como a mujeres mayores de 18 que hubieran consumido el producto y que tuvieran su domicilio en la capital del país, con el objetivo de obtener la caracterización de las tendencias de consumo; posteriormente, se llevó a cabo una observación a tiendas de barrio, supermercados y grandes superficies de la ciudad de Bogotá, con el fin de referenciar los procesos de distribución de este producto en el mercado.

Los resultados arrojados en esta investigación pretenden fortalecer no solo a la academia -desde la generación de nuevo conocimiento en relación con los conceptos de consumo y sus tendencias actuales, así como de las realidades mercadológicas de los canales de distribución para productos que pertenecen a la canasta familiar, como lo son las arepas de maíz-, sino también al sector industrial y comercial, en relación con la optimización de la toma de decisiones en beneficio del sector empresarial de la ciudad. Al respecto, desde la potencialidad de las arepas de maíz como producto de consumo masivo, sería pertinente para inversionistas privados el desarrollo de planes de negocio que permitan determinar el nivel de factibilidad de la producción y comercialización de este alimento, siendo el presente estudio un punto de partida frente a la identificación y perfilación de los diversos segmentos del mercado más favorables para esta industria y a la estructura de algunas actividades tácticas de marketing, como la gestión del producto, del precio y de su distribución.

El desarrollo de este estudio inicia con una revisión bibliográfica del marketing, su relacionamiento con los fenómenos de consumo y con los conceptos de distribución, generando así un soporte teórico de la investigación. Posteriormente, se presentan la metodología y sus resultados, incluyendo la discusión y las implicaciones para la caracterización de las tendencias de consumo y de los canales de distribución presentes en el mercado de la arepa de maíz en la ciudad de Bogotá, D. C.

\section{Marco teórico}

\section{La relevancia del mercadeo y su evolución}

La ciencia del mercadeo enmarca el tema general del presente estudio, de ahí la pertinencia de iniciar esta revisión resaltando su relevancia. Las últimas posturas que la Asociación Americana de Marketing (AMA) ha tenido frente al concepto de mercadeo 
tienen un común denominador: el valor que deben encontrar los stakeholders en las ofertas empresariales (López Rodríguez, 2016). La última postura de la AMA propone que "el marketing es la actividad, un conjunto de instituciones y procesos para crear, comunicar, entregar, y el intercambio de ofertas que tienen valor para los clientes, socios y la sociedad en general" (American Marketing Association, 2013).

Para McCarthy y Perreault (2001), el marketing es la realización de las actividades que pueden ayudar a que una empresa consiga las metas que se ha propuesto, anticipándose a los deseos de los consumidores, y a desarrollar productos o servicios aptos para el mercado. En complemento, "la mercadotecnia debe ser desarrollada por una empresa para identificar a los mercados meta, atraerlos, satisfacer sus necesidades o deseos y posicionarse en la mente de estos" (González González, Medina Quintero y Sánchez Limón, 2015, p. 15).

El marketing es relativamente reciente y desde su origen no ha dejado de evolucionar acorde con los tiempos y con los cambios que se han ido produciendo (Páramo, 2004). Anteriormente, los enfoques de las empresas se orientaban más a la producción que al mercado. Kotler y Keller (2006) mencionan que el enfoque de producción es uno de los más antiguos en el mundo de los negocios; en él, los consumidores favorecerán aquellos productos fáciles de conseguir y de bajo costo. Luego, el consumo y sus características generaron un cambio en el enfoque en que las organizaciones hacían las negociaciones: este enfoque era el de las ventas, el cual consistía en vender más cosas, a más gente, con mayor frecuencia, a cambio de más dinero, con el fin de conseguir mayores beneficios (Zyman, 2000). Con la llegada la globalización apareció otro enfoque organizacional conocido como el marketing relacional, el cual se direcciona a la continua entrega de valor, así como al desarrollo de ventajas competitivas sostenibles, de modo que se pueda hacer frente a nuevos competidores, lo anterior con el propósito de lograr diferenciación a largo plazo (Valle y Tobar, 2017).

Las empresas que apoyan el marketing relacional fomentan comunidades alrededor de su marca y de sus ofertas empresariales (López Rodríguez y Ortegón-Cortázar, 2017) e incrementan su visibilidad en el mercado. El mercadeo de relaciones es un proceso que consiste en manejar información detallada sobre clientes individuales, así como sobre los "puntos culminantes" en su relación con la empresa, con el fin de maximizar su fidelidad (Kotler y Keller, 2012). Por esta razón, en la medida en que las organizaciones se dieron cuenta de la importancia del mercado en el proceso económico, nació el marketing y posteriormente el marketing relacional (López Rodríguez, 2016).

El conocimiento que deben tener las organizaciones sobre sus clientes será un elemento diferenciador en términos comerciales, pues "el objetivo del marketing es conocer tan 
bien al cliente que el producto pueda ser definido o ajustado a sus necesidades de tal manera que se venda solo" (Braidot, 2002, p. 25); por ello, sin la existencia de marketing las empresas no hubieran evolucionado como lo han hecho, y aquellas que le han dado la espalda a la mercadotecnia difícilmente han conseguido sobrevivir (Martínez, 2011). Frente a lo anterior, en un ambiente mercadológico, la importancia de la dinámica demográfica durante el transcurso de los años llevó a una reinvención de estrategias y a una readaptación de las nuevas tendencias del consumo en los escenarios comerciales (Hernández y Vega, 2017). Actualmente es imperativo que las organizaciones posean en su filosofía corporativa una alta orientación al mercado, que debe ser un componente de la estrategia global de la organización que contribuirá de forma positiva a los resultados del negocio (Rojas y Vega, 2013). Por lo anterior, la presencia del mercadeo es tan relevante que debe observarse como una relación continua y constante de las organizaciones con los clientes, convirtiéndose así en una cultura organizacional para la empresa (López Rodríguez y Ortegón-Cortázar, 2017).

El marketing está conformado por componentes que deben estar claramente complementados a fin de solucionar problemas del mercado de acuerdo con sus propias realidades y en función de sus verdaderos deseos y expectativas. Para Valencia y Grillo (2013), estos componentes son:

El estudio del conocimiento, el análisis y la interpretación de los factores del entorno como los gustos, las preferencias, los deseos y las expectativas de los agentes del mercado desde la demanda organizacional empresarial que se impone a las restricciones del mercado.

Las estrategias y los programas de mercadeo que, en plena correspondencia con la realidad del entorno, se conciben para resolver problemas.

\section{Consumo como elemento del marketing}

Muchos estudios de marketing tienen como protagonista al consumo, no solo por el hecho de que las acciones de mercadeo se enfocan hacia su generación, sino por la participación de esta actividad como etapa final del proceso económico. Páramo (2004) manifiesta que el fenómeno del consumo es concebido como

[...] la conjugación y expresión de una serie de circunstancias propias del mundo moderno particularmente complejas y estrechamente relacionadas con el acto y la acción de adquisición y uso de productos, convirtiéndose en la columna vertebral del proceso de planeación e implementación del marketing [en el] interior de muchas organizaciones empresariales. (p. 223).

Desde la perspectiva de Guglielmucci (2015), la comprensión del consumo como una forma de comunicación permitió abordarlo como expresión de estatus o como un 
fenómeno capaz de construir una estructura de diferencias sociales; sin embargo, el consumo es una acción que se da de forma natural, sin importar la condición económica de los miembros de la sociedad, y se hace viable en función del poder adquisitivo de cada individuo (Otero y Giraldo, 2017). Los procesos de consumo deben observarse desde una perspectiva comercial, pero también desde una responsabilidad de las organizaciones; por ello, en beneficio de la sociedad, el consumo debe ser responsable en los ámbitos social y ambiental, debe cubrir las necesidades actuales de la oferta y la demanda, pero al mismo tiempo debe conservar o mejorar la capacidad de generaciones futuras para cubrir sus necesidades (Kotler y Armstrong, 2012).

Históricamente, los seres humanos han vivido en una sociedad de consumo, y como lo proponen Granada, Córdoba y Chaparro (2016), se debe entender el consumo como una acción cotidiana, lícita-placentera, en la cual se encuentran inmersos unos procesos psicológicos que inciden en el comportamiento y en la motivación del consumidor, pretendiendo satisfacer unas necesidades y unos deseos que confluyen entre la oferta, la demanda y los ideales sociales a los que se expone el comprador. Por esta razón, las personas "actúan y toman decisiones sobre los productos y servicios que adquieren en función de las percepciones que tengan de estos y de sus fabricantes y distribuidores" (León, 2008, p. 84).

Consumir es una operación cotidiana e imprescindible que está ligada a la reproducción material, cognitiva, emocional y sensorial de los individuos (Granada, Córdoba y Chaparro, 2016). De ahí la importancia del estudio de las experiencias del consumidor en términos de producto, compra y consumo propuesto por Ortegón-Cortázar y Gómez (2016), en el que cada una de ellas impacta directa e indirectamente el mercado, motivo por el cual debe suponerse un papel integrador de tales experiencias, tal como ocurre con el funcionamiento de los sentidos reportado por diversos estudios. Por ello, el comportamiento del consumidor se observa como el conjunto de actividades que realizan las personas cuando seleccionan, compran, evalúan y utilizan bienes y servicios con el objeto de satisfacer sus deseos y necesidades, actividades en las que están implicados procesos mentales y emocionales, así como acciones físicas (Mollá, Berenguer, Gómez y Quintanilla, 2014).

De acuerdo con Villalba (2005), el consumidor puede percibir de forma positiva o no los beneficios de la participación en una promoción, aspecto que fabricantes y detallistas deberán tener en cuenta a la hora de planificar sus acciones promocionales. Los consumidores forman parte de comunidades de marca, las cuales pueden realizar acciones en nombre de la marca que afecten de manera directa o indirecta la imagen y la reputación de esta (Pérez-Chavarría y Rodríguez-Ruiz, 2012), y muestran, cada vez más, mayor atención a los comportamientos de las empresas en relación con el medio 
ambiente, la producción limpia, el reciclaje, el abuso patronal y el enriquecimiento de empresas sin contrapartida a la sociedad (León, 2008). Así mismo, para Rivas (2004, p. 4), "el consumidor es una obligación actual de la empresa, en cuanto más se conozca se incrementarán las probabilidades de éxito empresarial” .

El consumidor actual ha venido evolucionando, y desde la perspectiva de Schiffman y Kanuk (2005) tiene más información que antes gracias a su participación en las comunidades virtuales, al crecimiento de ofertas de productos y a la digitalización de la información, que permite a los comerciantes personalizar los productos y servicios que ofrecen. Es necesario entender que las conductas de consumo son el resultado de un proceso psicológico, hedonista en la satisfacción de necesidades y deseos, en el que está implicada la cognición como un sistema de procesamiento de información que interacciona de forma constante con el mercado y que logra consolidar estereotipos o ideales que pueden ser permanentes en la mente del comprador (Granada, Córdoba y Chaparro, 2016). Así mismo, "los consumidores son más analíticos y consientes a la hora de decidir qué productos o servicios comprar, lo que puede de alguna forma evidenciarse en la aparición de movimientos de consumidores” (León, 2008, p. 89).

El consumo no solamente genera beneficios económicos, entendido este concepto como una variable macroeconómica. Desde una postura social, el fenómeno del consumo trae consigo una serie de beneficios que permean diversos escenarios en los cuales están inmersos los individuos. Al respecto, Warde (2000, citado en Páramo, 2004) relaciona los siguientes elementos como beneficios del consumo moderno: el consumo estimula el trabajo, promueve una actitud estética, facilita la expresión social, es agradable y placentero, nutre el individualismo competitivo, apoya prácticas socialmente significativas, provee estimulación intelectual, proporciona refrescante entretenimiento, estimula el confort y permite a cada uno obtener lo que desea. Los beneficiados de ello son los mercados, que, según Paris (2013), son "una población económicamente activa o pasiva conformada por grupos de consumidores organizacionales o finales" (p. 20).

A partir de la adquisición de bienes y servicios como conducta mercantil, el consumidor puede dar cuenta del interés que tiene frente a la marca y, a su vez, las industrias pueden interesarse por las conductas de consumo y los tipos de consumidores con el propósito de generar estrategias que puedan favorecer el mercado e incitar en el cliente una lealtad a la marca, de manera que se produzca en él un condicionamiento de su cognición y de sus emociones (Granada, Córdoba y Chaparro, 2016). Por ello, León (2008) resalta el hecho de que las empresas que estén sinceramente orientadas al consumidor, convencidas de que su existencia en el mercado depende de que los consumidores compren sus ofertas, tendrán que valorar y tener en cuenta la percepción de estos. 


\section{Relevancia del proceso de distribución}

Las relaciones con los canales de distribución, como uno de los stakeholders más relevantes para el crecimiento de las organizaciones, deben ser prioritarias para los empresarios debido a que "un productor que no es seleccionado por los intermediarios, que son sus principales compradores, corre el riesgo de perder espacios de mercado y encarar rápidas y sucesivas reducciones en sus ventas" (Hernández, Domínguez y Ramos, 2002, p. 146). Toro (2016) comenta que las formas de distribución comercial que utilizan las empresas han evolucionado y optimizado el uso de los recursos necesarios para realizar las operaciones logísticas de una manera mucho más eficiente, es decir, que a medida que pasa el tiempo las empresas tratan de implementar nuevas maneras de poder llegar con su producto al consumidor por el mejor medio posible.

Esta variable del marketing mix, como lo es la distribución, según Vásquez (2009, p. 10) “es un conjunto de organizaciones independientes comprometidas en el proceso de hacer que un producto o servicio esté disponible para su uso o consumo". Así mismo, puede observarse como una estructura de negocios y de organizaciones interdependientes que va desde el punto del origen del producto hasta el consumidor, la cual puede estar conformada por personas y compañías que intervienen en la transferencia de la propiedad de un producto a medida que este pasa del fabricante al consumidor final o al usuario industrial (Gómez, 2010). Para los intermediarios que conforman los diversos canales, "el propósito de contar con varios proveedores de manera simultánea se convierte en una ventaja por el efecto de la competencia entre los pequeños productores" (Hernández, Domínguez y Ramos, 2002, p. 147). Así mismo, Fernández, Martínez y Rebollo (2006) manifiestan que los canales "son un conjunto de empresas con relaciones de suministro o de distribución organizadas en torno a una mejor posición para dirigir y afrontar contingencias críticas que pueden encontrar en un mercado concreto los miembros de la red" (p. 190).

Para Paz (2008), la distribución es un proceso que conlleva actividades que se deben ejecutar para que el consumidor final pueda tener a su alcance el producto para poder comprarlo; además, en este proceso intervienen diferentes intermediarios que se relacionan entre sí para poder lograr el objetivo de esta actividad táctica del mercadeo. Los intermediarios de un canal cumplen funciones relevantes en el relacionamiento con los clientes, siendo estos mayoristas y minoristas. Al respecto, Sainz de Vicuña (2000) describe que los mayoristas compran los productos a los fabricantes o a otros mayoristas y los venden a los minoristas, pero nunca al consumidor final, mientras que los minoristas o detallistas compran las mercancías a los mayoristas o a los fabricantes y los venden directamente al consumidor final. 
Kotler y Keller (2012) proponen una clasificación de las estrategias de distribución de acuerdo con el número de intermediarios que se van a utilizar en el canal, estas estrategias son las siguientes:

- La distribución exclusiva, la cual consiste en limitar de forma importante el número de intermediarios para proporcionarle al fabricante control sobre el proceso de distribución de sus productos.

- La distribución selectiva, que consiste en la utilización de más de un intermediario, pero no de todos los que desean distribuir el producto en particular.

- La distribución intensiva, la cual consiste en la distribución de productos por medio de tantos puntos de venta como sea posible, en especial productos de la canasta familiar.

Hervás, Campo y Revilla (2012) mencionan que la distribución comercial se da cuando se contactan a productores y consumidores, lo cual hace necesario el traslado de productos del lugar de producción o fabricación al lugar de consumo, es decir, los fabricantes ponen a disposición de los consumidores sus productos mediante la distribución. Al respecto, en la figura 1 se proponen algunas formas de distribución comercial.

Figura 1. Formas de distribución comercial

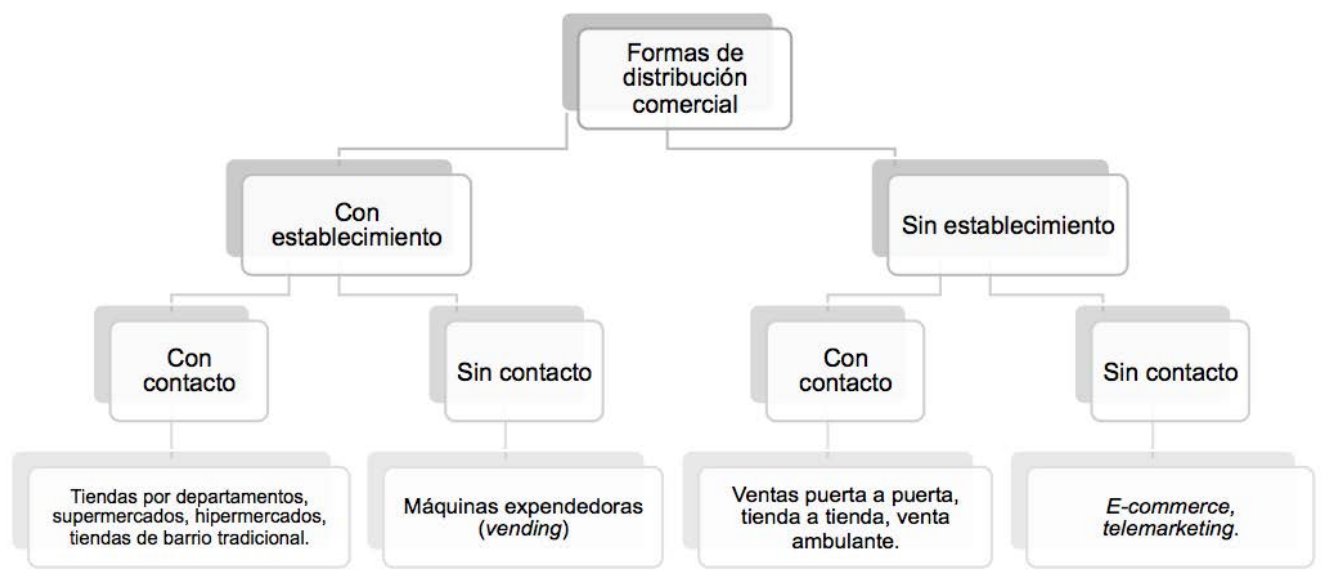


En el proceso de distribución, los diversos mercados existentes, entendidos como consumidores, poseen necesidades diversas. Al respecto, Nunes y Céspedes (2003) ubican a los compradores en las siguientes categorías en relación con los canales de marketing:

- Compradores habituales, los cuales compran en los mismos establecimientos y del mismo modo.

- Buscadores de las mejores ofertas, que conocen sus necesidades y "navegan" por los diversos canales antes de comprar al precio más bajo posible.

- Compradores amantes de la variedad, que recopilan información de numerosos canales, aprovechando los servicios de contacto humano, y después compran en su canal favorito, independientemente del precio.

- Compradores con altos niveles de implicación, los cuales reúnen información de todos los canales, compran en el que les ofrece el mejor precio, pero aprovechan los servicios al cliente de los canales con contacto personal.

Desde lo anterior, el relacionamiento de los canales con los clientes potenciales es muy imperativo respecto a la lealtad del consumidor con la propuesta de valor de las organizaciones, de ahí la importancia de visualizar las funciones propias del canal de distribución en función del consumo. Al respecto, la tabla 1 relaciona estas funciones del canal de marketing.

\section{Tabla 1. Funciones del canal de marketing}

\section{Funciones del canal de marketing}

Recopilar información sobre clientes reales y potenciales, competidores y demás agentes y fuerzas del entorno de marketing.

Desarrollar y distribuir comunicaciones persuasivas para estimular las ventas.

Firmar acuerdos de precio y demás condiciones para traspasos de propiedad o posesión.

Hacer pedidos a los fabricantes.

Conseguir los fondos necesarios para financiar inventarios a diferentes niveles del canal de marketing.

Asumir riesgos vinculados con el desarrollo del trabajo del canal.

Facilitar el almacenamiento y el flujo sucesivo de los productos físicos.

Ofrecer facilidades de pago a los compradores por medio de diversas líneas y alternativas.

Supervisar la transferencia de posesión real de una persona u organización a otra.

Fuente: Elaboración propia con base en Kotler y Keller (2012). 


\section{Metodología}

De acuerdo con la literatura revisada y el objetivo de estudio propuesto, esta investigación se abordó desde dos perspectivas metodológicas. En la primera parte, el trabajo de campo desarrolló un diseño descriptivo transversal, puesto que se persiguió detallar las diferentes características que poseen los consumidores de arepas de maíz en la ciudad de Bogotá, D. C. en un único periodo de tiempo. Los estudios descriptivos "miden o evalúan diversos aspectos, dimensiones o componentes del fenómeno o fenómenos [que se van] a investigar” (Hernández, Fernández y Baptista, 2010, p. 61). Así mismo, el método fue cuantitativo, por ello se usaron técnicas de carácter descriptivo por medio de las cuales se determinaron "cuantos" o con qué "frecuencia" ocurre un determinado proceso (Martínez-Carazo, 2006). De igual forma, se utilizó un muestreo no probabilístico por conveniencia; de acuerdo con Torres, Paz y Salazar (2006), este tipo de muestreo le permite al investigador seleccionar directa e intencionadamente a los individuos de la población de su estudio. La unidad de información estuvo compuesta por hombres y mujeres mayores de 18 años, residentes en la ciudad de Bogotá y que usualmente hubieran consumido el producto estudiado, lo que se verificó en las preguntas filtro del instrumento. El instrumento se aplicó durante septiembre y octubre de 2016. Para el estudio se estimó una muestra de 384 personas con un nivel de confianza del $95 \%$, un coeficiente de variación positiva y negativa del $50 \%$ cada uno y un margen de error del $5 \%$.

En segunda instancia, se desarrolló un proceso cualitativo denominado inmersión en el contexto, mediante una observación y una bitácora de campo. En este proceso se identificaron los tipos de datos que se iban a recolectar, la muestra de a quién(es) observar, el cuándo y el dónde recolectar los datos, el tiempo de aplicación y la definición del rol del investigador (Hernández, Fernández y Baptista, 2010, p. 382). Teniendo en cuenta la estructura de inmersión en el contexto, se aplicaron la observación y las bitácoras de campo a veinte (20) tiendas de barrio, diez (10) supermercados y tres (3) grandes superficies ubicadas en la ciudad de Bogotá durante noviembre de 2016. En estos instrumentos se registraron elementos de los canales de distribución, entre los cuales sobresalen su formato y/o tipología y el precio de los productos. Esta bitácora de observación es un instrumento de recolección de datos que acompaña al observador de campo y cuya función consiste en guardar de forma primaria, y así como se presentan, todos los datos que se consideran pertinentes para el tema de una investigación; es un cuaderno o diario en el que se registra con la mayor fidelidad posible lo que se observa de la realidad desde las apreciaciones del observador, las emociones y las reacciones del objeto de estudio (Krumm, 2007, p. 2). 


\section{Resultados}

Es relevante tener en cuenta que "las prácticas relacionadas con la alimentación han interesado a las personas, y con el tiempo, en diferentes sitios geográficos, la alimentación trasciende las vidas en los hogares, en las relaciones sociales y en cada lugar donde las personas se relacionan” (Melchor, Rodríguez y Díaz, 2016, p. 177). Frente a ello, desde el instrumento aplicado a la muestra establecida, se observan las principales tendencias de consumo del mercado de las arepas de maíz en la ciudad de Bogotá, D. C.

Figura 2. Sitios donde se consume arepa de maíz por edades

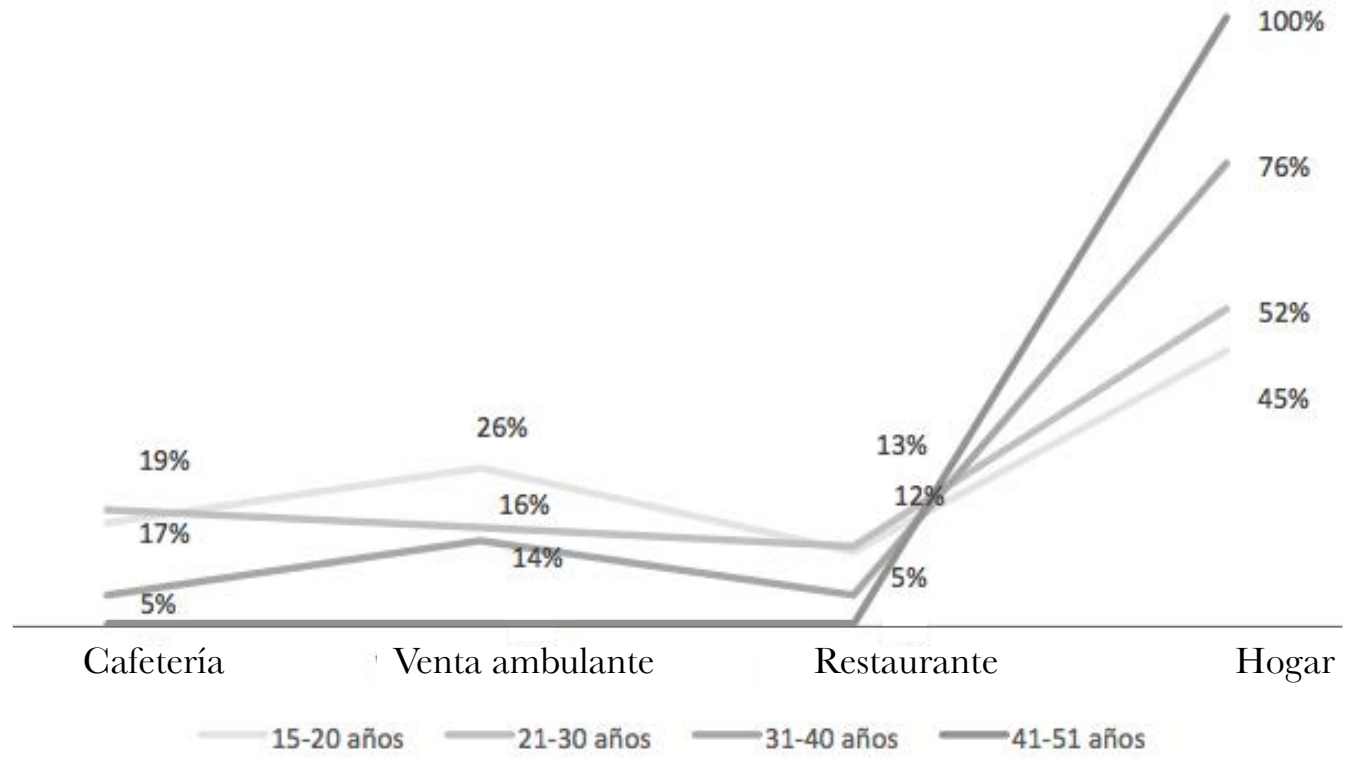

Fuente: Elaboración propia.

El estudio evidencia que el $100 \%$ de las personas mayores de 41 años consumen en el hogar el producto estudiado; por ello, en este segmento demográfico de la población, la familia y los aspectos culturales son factores que influyen en la decisión de compra y consumo (Melchor, Rodríguez y Díaz, 2016). El 56 \% de la población entre los 15-20 años consumen arepas de maíz fuera de casa, así como el $48 \%$ de la población entre 21-30 años, siendo una fracción del mercado importante para que las empresas que distribuyen el producto a minoristas tengan en cuenta las cafeterías, los restaurantes y las ventas ambulantes como canales relevantes. 
Figura 3. Razones para consumir arepas de maíz por estrato

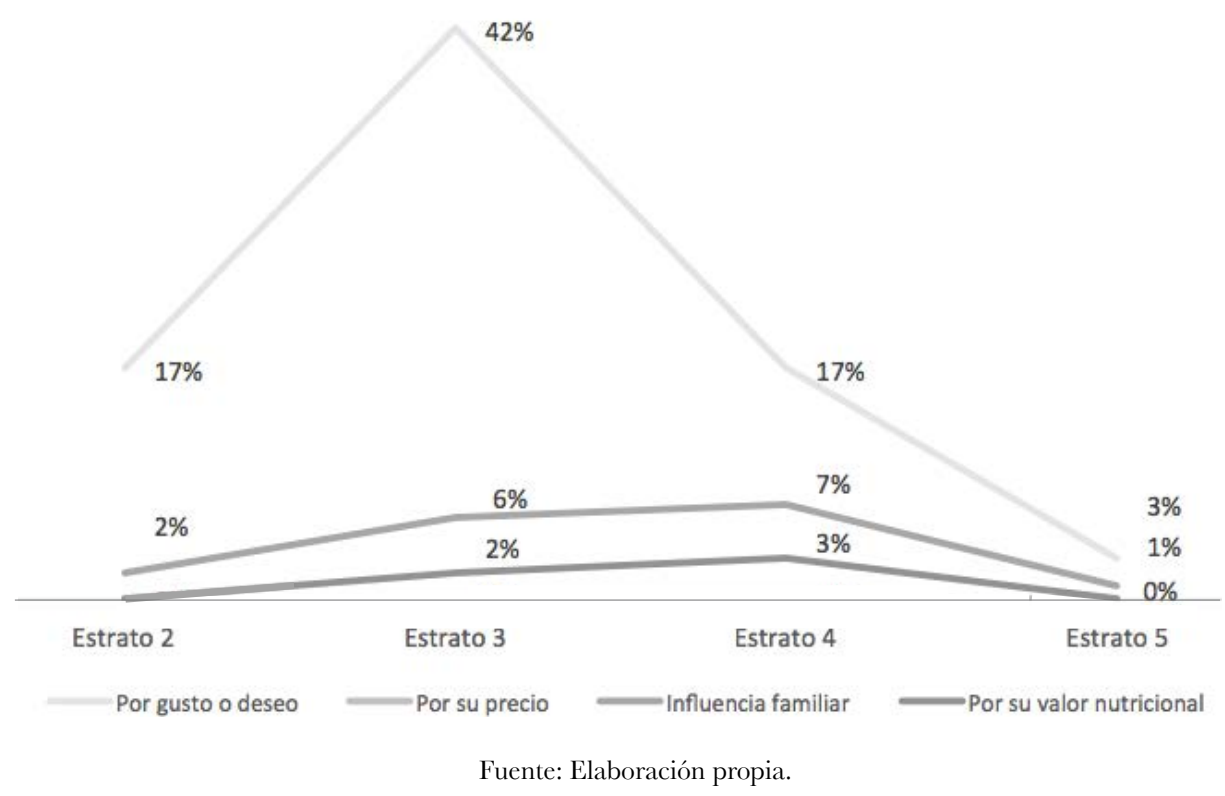

El trabajo de campo determina que la razón principal para consumir arepas de maíz en los diferentes estratos es por gusto o deseo, con el $79 \%$; le siguen la influencia familiar $(16 \%)$ y el valor nutricional $(5 \%)$ en la influencia de compra. La variable con menor participación es el precio, generando así una característica particular de las tendencias de consumo de la población estudiada en relación con esta variable del marketing mix.

Figura 4. Razones para consumir arepa de maíz por estrato

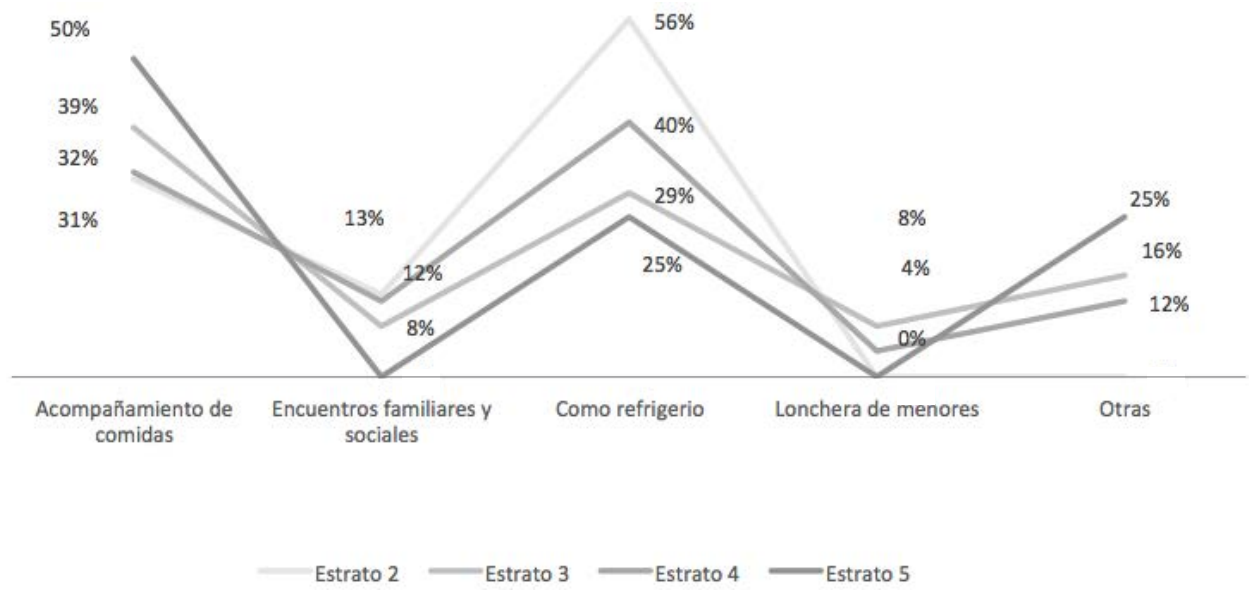

Fuente: Elaboración propia. 
En cuanto al consumo de arepas de maíz por estrato, la información recolectada evidencia que el usar el producto como acompañamiento de comidas y el considerarlo como refrigerio son las principales razones para su consumo; estos dos elementos son tendencias que deben tener en cuenta los diversos canales de distribución para optimizar su propuesta de valor en beneficio de los usuarios finales del producto, tal como lo afirman Kotler y Keller (2012) cuando describen que los canales de distribución deben ser conjuntos de organizaciones que participan en el proceso de poner a disposición de los consumidores un bien o servicio para su uso o adquisición.

Figura 5. Canales de distribución donde más se adquieren arepas de maíz por estrato

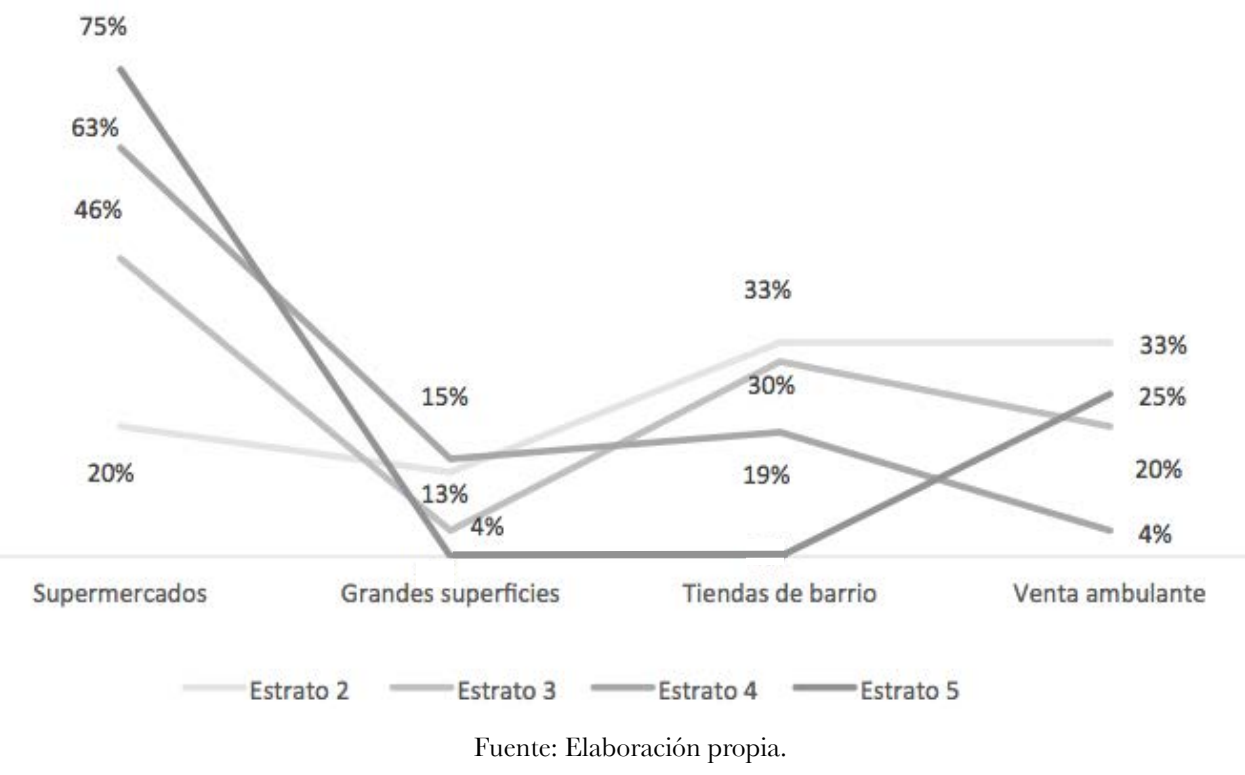

La información obtenida muestra que los supermercados son el canal de distribución más utilizado por los segmentos de mercado pertenecientes a los estratos 3, 4 y 5 . La mayor distribución del producto en el estrato 2 la tienen las ventas ambulantes. Por ello, la importancia de la distribución en beneficio no solo del consumidor, sino también de las organizaciones, es el alcance de la estructura de un sistema de canales de marketing, el cual tiene como principal objetivo lograr que los consumidores potenciales realicen pedidos rentables, pues estos canales no solo deben atender mercados, sino también deben crearlos (Kotler y Keller, 2012).

\section{Comportamiento de los canales de distribución}

De acuerdo con las bitácoras de observación, los principales canales de distribución del mercado de las arepas de maíz en esta investigación fueron agrupados en tres (3) 
grandes categorías, en cada una de las cuales existe una oferta de diferentes variedades de productos que se relacionan a continuación:

Tabla 2. Relación de tipologías del producto en las tres (3) categorías de minoristas

\begin{tabular}{|c|c|c|c|}
\hline \multicolumn{2}{|c|}{ Grandes superficies } & \multirow{2}{*}{$\begin{array}{c}\text { Supermercados } \\
\text { Antioqueña }\end{array}$} & \multirow{2}{*}{$\begin{array}{l}\text { Tiendas de barrio } \\
\text { Antioqueña grande }\end{array}$} \\
\hline Amarilla con sal & Integrales & & \\
\hline Amarilla sin sal & Maíz sancochado & Queso y mantequilla & Antioqueña pequeña \\
\hline Antioqueña & Pincho & Maíz peto & \multirow[t]{6}{*}{ Rellena de queso } \\
\hline Blanca con sal & Queso campesino & Maíz peto y sal & \\
\hline Blanca sin sal & Queso doble crema & Maíz peto, queso & \\
\hline Choclo & Queso mozzarella & Choclo & \\
\hline Boyacense & Santandereana & Blanca con sal & \\
\hline Extradelgada & Tela & Rellena de queso & \\
\hline
\end{tabular}

Se observa que en las categorías de los diferentes canales de distribución existen más de treinta variedades de arepas de maíz. En las categorías "grandes superficies" y "supermercados", estos productos deben estar registrados ante el INVIMA y otras entidades de regulación legislativa. En la categoría "tiendas de barrio" estas normas legales no son controladas ni exigidas por estos minoristas, de ahí la gran proliferación de fábricas familiares con baja capacidad de producción y bajos precios al mercado; sin embargo, estos "negocios" tienen cierta participación en el mercado informal.

\section{Análisis de los precios}

Para analizar los elementos más relevantes en la determinación del precio de las arepas de maíz en el mercado de Bogotá, se determinó un precio promedio en cada una de las cuatro categorías estudiadas y cada uno de los tipos de arepas que en ellas se están ofreciendo cotidianamente.

\section{Grandes superficies}

Tomando una muestra de tres (3) formatos que pertenecen a esta categoría, los resultados del análisis de la observación del precio son los siguientes: 
Tendencias de consumo y de la distribución en el mercado de las arepas de maíz en la ciudad de Bogotá, D. C.

Tabla 3. Precios promedios de las arepas en grandes superficies

\begin{tabular}{|c|c|c|c|}
\hline Marca & Descripción & Cantidad & Precio \\
\hline \multirow{4}{*}{ Delicias de Maíz } & Santandereana & 6 unid. & $\$ 6250$ \\
\hline & Antioqueña & 10 unid. & $\$ 3890$ \\
\hline & Queso y mantequilla & 5 unid. & $\$ 5890$ \\
\hline & Doble crema & 20 unid. & $\$ 8220$ \\
\hline \multirow{8}{*}{ Don Maíz } & Con sal & 8 unid. & $\$ 3780$ \\
\hline & Extradelgada & 15 unid. & $\$ 5370$ \\
\hline & Aparrillada & 5 unid. & $\$ 8350$ \\
\hline & Choclo & 5 unid. & $\$ 6610$ \\
\hline & Queso y mantequilla & 6 unid. & $\$ 6150$ \\
\hline & Arepa pequeña & 10 unid. & $\$ 4890$ \\
\hline & Antioqueña & 10 unid. & $\$ 6250$ \\
\hline & Súper especial queso & 5 unid. & $\$ 4780$ \\
\hline \multirow{4}{*}{ Marca propia } & Santandereana & 5 unid. & $\$ 4610$ \\
\hline & Arepa antioqueña & 10 unid. & $\$ 4290$ \\
\hline & Queso & 5 unid. & $\$ 4190$ \\
\hline & Queso doble crema & 6 unid. & $\$ 5010$ \\
\hline \multirow{6}{*}{ Doña Paisa } & Tela blanca & 10 unid. & $\$ 3550$ \\
\hline & Tela amarilla & 5 unid. & $\$ 3690$ \\
\hline & Con sal & 5 unid. & $\$ 3890$ \\
\hline & Mantequilla & 5 unid. & $\$ 3350$ \\
\hline & Rellena de queso & 5 unid. & $\$ 6680$ \\
\hline & Para pincho & 10 unid. & $\$ 3200$ \\
\hline \multirow{10}{*}{ Gransoli } & Integral & 5 unid. & $\$ 3790$ \\
\hline & Blanca con sal & 10 unid. & $\$ 3300$ \\
\hline & Blanca sin sal & 10 unid. & $\$ 3250$ \\
\hline & Amarilla con sal & 10 unid. & $\$ 3300$ \\
\hline & Amarilla sin sal & 10 unid. & $\$ 3400$ \\
\hline & Antioqueña & 10 unid. & $\$ 3820$ \\
\hline & Baja en grasa & 5 unid. & $\$ 3390$ \\
\hline & Rellena de queso & 6 unid. & $\$ 6410$ \\
\hline & Integral & 5 unid. & $\$ 3990$ \\
\hline & Pincho & 10 unid. & $\$ 3790$ \\
\hline \multirow{2}{*}{ Santa Bárbara } & Blanca con sal & 10 unid. & $\$ 3990$ \\
\hline & Rellena de queso & 10 unid. & $\$ 7090$ \\
\hline
\end{tabular}

Fuente: Elaboración propia con base en el trabajo de campo realizado en noviembre de 2016. 
Se puede analizar que la gama de precios es amplia y cambia de acuerdo con la variedad y el tipo de arepa. La más costosa es ofrecida por la empresa Don Maíz, con un precio de $\$ 8350$, presentación de 5 unidades (aparrillada); y la más económica es ofrecida por la empresa Doña Paisa, con un precio de $\$ 3200$ y presentación de 10 unidades (para pincho). Claramente se evidencia una segmentación de precios de acuerdo con las marcas y los beneficios ofrecidos por el producto.

\section{Supermercados}

Tomando una muestra de diez (10) formatos que pertenecen a esta categoría, los resultados obtenidos en el análisis de la observación del precio son los siguientes:

Tabla 4. Precios promedios de las arepas en supermercados

\begin{tabular}{|c|c|c|c|}
\hline Marca & Descripción & Cantidad & Precio \\
\hline \multirow{3}{*}{ Comestibles Yiyi } & \multirow{2}{*}{ Maíz peto y sal } & 8 unid. & $\$ 3800$ \\
\hline & & 10 unid. & $\$ 3100$ \\
\hline & Maíz peto, queso & 5unid. & $\$ 3800$ \\
\hline \multirow{3}{*}{ Doña Leidy } & \multirow{3}{*}{ Maíz peto } & 5 unid. & $\$ 3200$ \\
\hline & & 5 unid. & $\$ 3000$ \\
\hline & & 10 unid. & $\$ 4200$ \\
\hline Arepas Petos & Maíz choclo & 5 unid. & $\$ 4650$ \\
\hline El Cacique & Maíz precocido con queso & 5 unid. & $\$ 4650$ \\
\hline \multirow{3}{*}{$\begin{array}{c}\text { Productos y Arepas de Mi } \\
\text { Tierra Ltda. }\end{array}$} & Arepas integrales & 4 unid. & $\$ 4200$ \\
\hline & Arepa blanca & 10 unid. & $\$ 4400$ \\
\hline & Arepas rellenas de queso & 4 unid. & $\$ 5250$ \\
\hline \multirow{2}{*}{ Don Maíz } & Antioqueña & 6 unid. & $\$ 5400$ \\
\hline & Queso y mantequilla & 4 unid. & $\$ 4150$ \\
\hline \multirow{2}{*}{ Doña Paisa } & Tela blanca & 10 unid. & $\$ 4650$ \\
\hline & Rellena de queso & 4 unid. & $\$ 4990$ \\
\hline
\end{tabular}

Fuente: Elaboración propia con base en el trabajo de campo realizado en noviembre de 2016.

Se observa que los precios en esta categoría son inferiores a los de la categoría grandes superficies. La más costosa es la arepa antioqueña de Don Maíz, con un precio de $\$ 5400$, y la más económica es la arepa de maíz peto de Doña Leidy, con un precio de $\$ 3000$.

\section{Tiendas de barrio}

Tomando una muestra de 20 tiendas de barrio, se observa que los precios son muy equilibrados y van de acuerdo con el tamaño y la variedad del producto. 
Tabla 5. Precios promedios de las arepas en tiendas de barrio

\begin{tabular}{ccc}
\hline Descripción & Cantidad & Precio \\
\hline Antioqueña grande & 10 unid. & $\$ 4000$ \\
\hline Antioqueña pequeña & 5 unid. & $\$ 3400$ \\
Rellena de queso & 10 unid. & $\$ 3500$ \\
Fuente: Elaboración propia con base en el trabajo de campo realizado en noviembre de 2016.
\end{tabular}

\section{Conclusiones}

Las tendencias de consumo del producto investigado en este documento determinan que los estratos 2 y 3 son los segmentos del mercado que más consumen el producto analizado. Lo anterior identifica que las variables socioeconómicas conforman un elemento relevante para los procesos tácticos de las empresas pertenecientes al sector y convierten la diferenciación del producto, los precios y la distribución en determinantes del comportamiento del consumidor (Stanton, Etzel y Walker, 2004).

La edad de los consumidores como elemento demográfico determina que la población menor de 30 años consume arepas de maíz de preferencia fuera de casa, principalmente en las cafeterías, restaurantes y ventas ambulantes, a diferencia de las personas con una edad superior a los 31 años, quienes en su gran mayoría consumen el producto en el hogar, de preferencia como acompañamiento de comidas, y este es adquirido en supermercados. De esta manera, este segmento del mercado abarca a los consumidores que adquieren las arepas de maíz en los diferentes puntos minoristas y/o detallistas, de forma que se convierte en el mercado objetivo de mayor relevancia para los miembros de este tipo de canal de distribución, el cual debe proporcionar a los consumidores la información necesaria en cuanto a sus ofertas en diversos canales de comunicación (Schiffman y Kanuk, 2005).

Luego de hacer un análisis de los productos en los diversos canales de distribución, se observa que en el mercado existe una amplia y variada gama de sus precios de acuerdo con la referencia y la marca de las arepas de maíz; sin embargo, los resultados evidencian que esta variable no posee una fuerte influencia en el consumo de los mismos productos, como sí la tienen el gusto o deseo y la influencia familiar que tienen los consumidores por la arepa de maíz. Desde lo anterior, se sustentan algunos de los beneficios del consumo moderno planteados por Warde (2000, citado en Páramo, 2004), como el hecho de que el consumo es agradable y placentero, permitiendo a cada uno obtener lo que desea. 
Los hallazgos de la investigación permiten identificar que, para los estratos 4 y 5, los canales de distribución donde más se compran arepas de maíz son los supermercados. Por otra parte, las tiendas de barrio son el canal más relevante para adquirir el producto por parte de los estratos 2 y 3 . Lo anterior permite concluir que la familia capitalina, independientemente de su estrato socioeconómico, sí consume el producto. Por tal razón, resulta entonces importante para las empresas reconocer los canales adecuados de acuerdo con su segmento de mercado objetivo y determinar de manera asertiva sus estrategias comerciales para cada uno de ellos. Por ello, "el éxito de las estrategias de mercado dependerá en gran medida en la adecuada caracterización de estos segmentos objetivo que han sido identificados" (Caicedo Lamprea, Montoya Restrepo y Montoya Restrepo, 2015, p. 15). De ahí que el relacionamiento con los canales de distribución es trascendental para el fortalecimiento comercial de los fabricantes del producto, pues si alguno de ellos no es seleccionado por los miembros del canal, corre el riesgo de disminuir sustancialmente su participación en el mercado (Hernández, Domínguez y Ramos, 2002. p. 146).

Los hallazgos encontrados en el presente estudio, de forma consecuente con la revisión de la literatura, permiten reconocer algunas tendencias de consumo del producto estudiado mencionadas anteriormente, así como la participación de los canales de distribución más usados por estos segmentos del mercado. Sin embargo, una de las limitaciones de la investigación fue el tamaño de la muestra en la segunda etapa de la estrategia metodológica, en la que se aplicó la observación a estos canales. Se establece como alternativa metodológica en futuras investigaciones desarrollar un análisis longitudinal, mas no transversal, de tal forma que se puedan identificar rasgos de las tendencias de consumo de los mismos segmentos en diferentes periodos de tiempo.

\section{Referencias}

American Marketing Association. (2013). Definition of marketing. Recuperado de https:// www.ama.org/AboutAMA/Pages/Definition-of-Marketing.aspx

Ares, B. y Brenes, P. (2014). Dinamización del punto de venta. Madrid: Editex.

Braidot, N. P. (2002). Nuevo marketing total. Madrid: McGraw-Hill e IFEMA.

Caicedo Lamprea, S., Montoya Restrepo, L. A. y Montoya Restrepo, I. A. (2015). Propuesta de posicionamiento y segmentación de la carne de pavo (Meleagris gallopavo) en el entorno colombiano. Una aproximación desde el caso bogotano. Poliantea, 11(21), 13-38. DOI: 10.15765/plnt.v1 1i21.702

Fernández, Á., Martínez, E. y Rebollo, A. (2006). La nueva configuración de los canales de distribución: el comercio de electrodomésticos. Revista ICE, 828, 187-202. 
Recuperado de http://www.revistasice.com/CachePDF/ICE_828_187-202_62FA F7E389AA61CF61FE17CE7598B3A4.pdf

Gómez, A. (2010). Canales de distribución. Cali: Universidad ICESI.

González González, M., Medina Quintero, J. M. y Sánchez Limón, M. L. (2015). Las redes sociales: herramienta de mercadotecnia para el sector restaurantero. Poliantea, 11(20), 13-36. DOI: 10.15765/plnt.v1 1i20.650

Granada, L., Córdoba, D. y Chaparro, L. (2016). Aproximación a la comprensión de las conductas de consumo desde el marco jurídico colombiano y psicosocial. Funlam Fournal of Students' Research (FSR), 1, 139-147. DOI: 10.21501/25007858.2149

Guglielmucci, A. (2015). Publicidad, antropología y etnografía del consumo: coqueteos actuales entre disciplinas divergentes. Poliantea, 11(21), 41-58. DOI: 10.15765/plnt. v11i21.703

Hernández, P. y Vega, H. (2017). Cambio demográfico y consumo. La importancia del macroambiente en las estrategias mercadológicas. Fóvenes en la Ciencia, 2(1), 725-729.

Hernández, J., Domínguez, M. L. y Ramos, A. O. (2002). Canales de distribución y competitividad en artesanías. Espiral. Estudios sobre Estado y Sociedad, 9(25), 143164. Recuperado de http://www.espiral.cucsh.udg.mx/index.php/EEES/article/ view/1242/1123

Hernández, R., Fernández, C. y Baptista, M. (2010). Metodología de la investigación (5. ${ }^{\text {a ed.). }}$ México, D. F.: McGraw-Hill. Recuperado de goo.gl/uxuUWC

Hervás, A., Campo, A. y Revilla, M. (2012). Animación del punto de venta. Madrid: McGraw-Hill.

Kotler, P. y Armstrong, G. (2012). Marketing (14. ${ }^{\mathrm{a}}$ ed.). México, D. F.: Pearson Educación de México, S. A. de C. V. Recuperado de https://profdariomarketing.files.wordpress. com/2014/03/marketing_kotler-armstrong.pdf

Kotler, P. y Keller, K. (2006). Dirección de marketing (12. ${ }^{a}$ ed.). México, D. F.: Pearson Educación de México, S. A. de C. V.

Kotler, P. y Keller, K. (2012). Dirección de marketing (14.a ed.). México, D. F.: Pearson Educación de México, S. A. de C. V. Recuperado de https:/ / asesoresenturismoperu. files.wordpress.com/2016/05/182-direccion-de-marketing-philip-kotler.pdf

Krumm, N. S. (2007). La bitácora de recolección de datos. Montemorelos, N. L.: Centro de Recursos para la Enseñanza y el Aprendizaje (CREA) de la Universidad de Montemorelos. 
León, F. (2008). La percepción de la responsabilidad social empresarial por parte del consumidor. Visión Gerencial, 7(1), 83-95. Recuperado de http://www.saber.ula.ve/ bitstream/123456789/25179/2/articulo6.pdf

López Rodríguez, C. E. (2016). El gerente competitivo y su rol en las mipymes: una perspectiva desde el marketing. Equidad y Desarrollo, 25, 209-224. DOI: 10.19052/ed.3726

López Rodríguez, C. E. y Ortegón-Cortázar, L. (2017). Del marketing político a las comunidades de marca. Un estudio comparativo de partidos políticos en Bogotá, D. C. Universidad \& Empresa, 19(32), 9-35. Recuperado de http://revistas.urosario.edu.co/ index.php/empresa/article/view/4552

Martínez-Carazo, P. (2006). El método de estudio de caso: estrategia metodológica de la investigación científica. Pensamiento y Gestión, 20, 165-193. Recuperado de http:// www.redalyc.org/pdf/646/64602005.pdf

Martínez, J. A. (2011). Evolución del marketing: desde el egocentrismo a la orientación al consumidor. Contribuciones a la Economía, (diciembre). Recuperado de http://www. eumed.net/ce/2011b/jamg2.html

McCarthy, E. J. y Perreault, W. D. (2001). Marketing: un enfoque global (13. a ed.). México, D. F.: McGraw-Hill.

Melchor, M., Rodríguez, J. D. y Díaz, M. A. (2016). Comportamiento de compra y consumo de productos dietéticos en los jóvenes universitarios. Pensamiento \& Gestión, 41, 174-193. DOI: 10.14482/pege.41.9709

Mollá, A. (Coord.), Berenguer, G., Gómez, M. A. y Quintanilla, I. (2014). Comportamiento del consumidor (1. a ed. digital). Barcelona: Editorial UOC. Recuperado de http://reader.digitalbooks.pro/book/preview/28750/Section0003.htm?1511977992790

Nunes, P. y Céspedes, F. (2003). The customer has escaped. Harvard Business Review, 81(11), 96-105. Recuperado de https://hbr.org/2003/11/the-customer-has-escaped

Ortegón-Cortázar, L. y Gómez, A. G. (2016). Gestión del marketing sensorial sobre la experiencia del consumidor. Revista de Ciencias Sociales (Ve), 22(3), 67-83. Recuperado de http://www.redalyc.org/articulo.oa?id=28049146006

Otero, M. C. y Giraldo, W. (2017). Consumo de productos infantiles en la base de la pirámide poblacional: análisis de los mecanismos influyentes. Económicas $C U C, 38(1)$, 165-184. DOI: 10.17981/econcuc.38.1.08 
Páramo, D. (2004). El fenómeno de consumo y el consumo en marketing. Convergencia. Revista de Ciencias Sociales, 11(34), 221-250. Recuperado de http://www.redalyc.org/ articulo.oa?id=10503409

París, J. A. (2015). Aplicación del cuadrado de Greimas a los cuatro aspectos esenciales del marketing. Poliantea, 11(21), 61-102. DOI: 10.15765/plnt.v1 1i21.704

París, J. (2013). Hacia la segmentación significativa. Poliantea, 9(17), 11-39. DOI: g/10.15765/plnt.v9i17.462

Paz, H. R. (2008). Canales de distribución. Gestión comercial y logística (1. ${ }^{\text {a }}$ ed.). México, D. F.: Lectorum Ugerman.

Pérez-Chavarría, M. y Rodríguez-Ruiz, A. (2012). Comunidades de marca: otra manera de sostener conversaciones y generar relaciones públicas. Revista Internacional de Relaciones Públicas, 2(3), 175-192. Recuperado de http://revistarelacionespublicas. uma.es/index.php/revrrpp/article/viewFile/78/67

Peris, S. M., Parra, F., Lhermie, G. y Romero, M. A. (2008). Distribución comercial (6. ${ }^{\text {a }}$ ed., revisada y actualizada). Madrid: ESIC Editorial.

Portafolio. (2016). Siete de cada 10 colombianos consumen arepa en el desayuno. Portafolio [en línea]. Recuperado de http://www.portafolio.co/negocios/empresas/ siete-de-cada-10-colombianos-consumen-arepa-en-el-desayuno-498533

Rebollo, A. y Casares Ripol, J. (2002). La innovación en la distribución comercial. El papel de MERGASA y la Red de MERGAS. Distribución y Consumo, 66, 5-23.

Rivas, J. A. y Grande, I. (2004). Comportamiento del consumidor. Decisiones y estrategia de marketing (5. ${ }^{\text {a }}$ ed., revisada y actualizada). Madrid: ESIC.

Rojas, S. P. y Vega, R. A. (2013). Uso del mercadeo estratégico en las mipymes de Bogotá. Poliantea, 7(13), 97-111. DOI: 10.15765/plnt.v7i13.142

Sainz de Vicuña Ancín, J. M. (2000). La distribución comercial: opciones estratégicas. Madrid: ESIC Editorial.

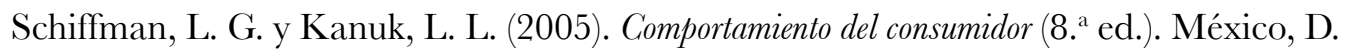
F.: Pearson Educación.

Stanton, W., Etzel, M. y Walker, B. (2004). Fundamentos de marketing (13. a ed.). México: McGraw-Hill Interamericana. 


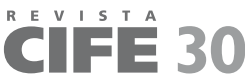

ISSN: 0124-3551 / Año 19, n. 30 / enero-junio / pp. 149-172

Toro, Á. (2016). Formas de distribución comercial de las empresas en la actualidad y su influencia en el consumidor final. Recuperado de http://repositorio.utmachala.edu.ec/bitstream/48000/7969/1/ECUACE-2016-AE-CD00085.pdf

Torres, M., Paz, K. y Salazar, F. (2006). Tamaño de una muestra para una investigación de mercado. Boletín Electrónico, Universidad Rafael Landivar, 2(02), 1-13. Recuperado de goo.gl/EFYW6j

Valle, A. y Tobar, G. (2017). El marketing relacional y la rentabilidad en los servicios prestados por tres concesionarios automotrices en la provincia de Tungurahua del Ecuador. Uniandes Episteme, 4(2), 135-149. Recuperado de http://186.46.158.26/ojs/ index.php/EPISTEME/article/view/489/268

Valencia, H. y Grillo, C. (2013). Sistema de gestión de marketing para las organizaciones solidarias en la ciudad de Bogotá, D. C. (Tesis de maestría). Universidad de Manizales, Manizales.

Vásquez, G. (2009). Los canales de distribución y el valor para el consumidor. Temas de Management, 2, 10-16. Recuperado de goo.gl/YfonjX

Villalba, F. J. (2005). La promoción de ventas y los beneficios percibidos por el consumidor. Revista Europea de Dirección y Economía de la Empresa, 14(3), 209-222.

Zyman, S. (2000). El fin del marketing tal y como lo conocemos. Madrid. Hapercollins. 